\title{
Auswirkungen desinfizierender UV-C-Bestrahlung testen
}

\author{
Zur Eindämmung der aktuellen Corona-Pandemie kommt zur Desinfektion zunehmend \\ UV-C-Strahlung zum Einsatz. Jedoch sind die Auswirkungen der Strahlung auf Materialien \\ und Beschichtungen häufig unbekannt. Hier unterstützen UV-C-Prüfgeräte die Verbesserung \\ der Methoden zur Testung von Beschichtungen.
}

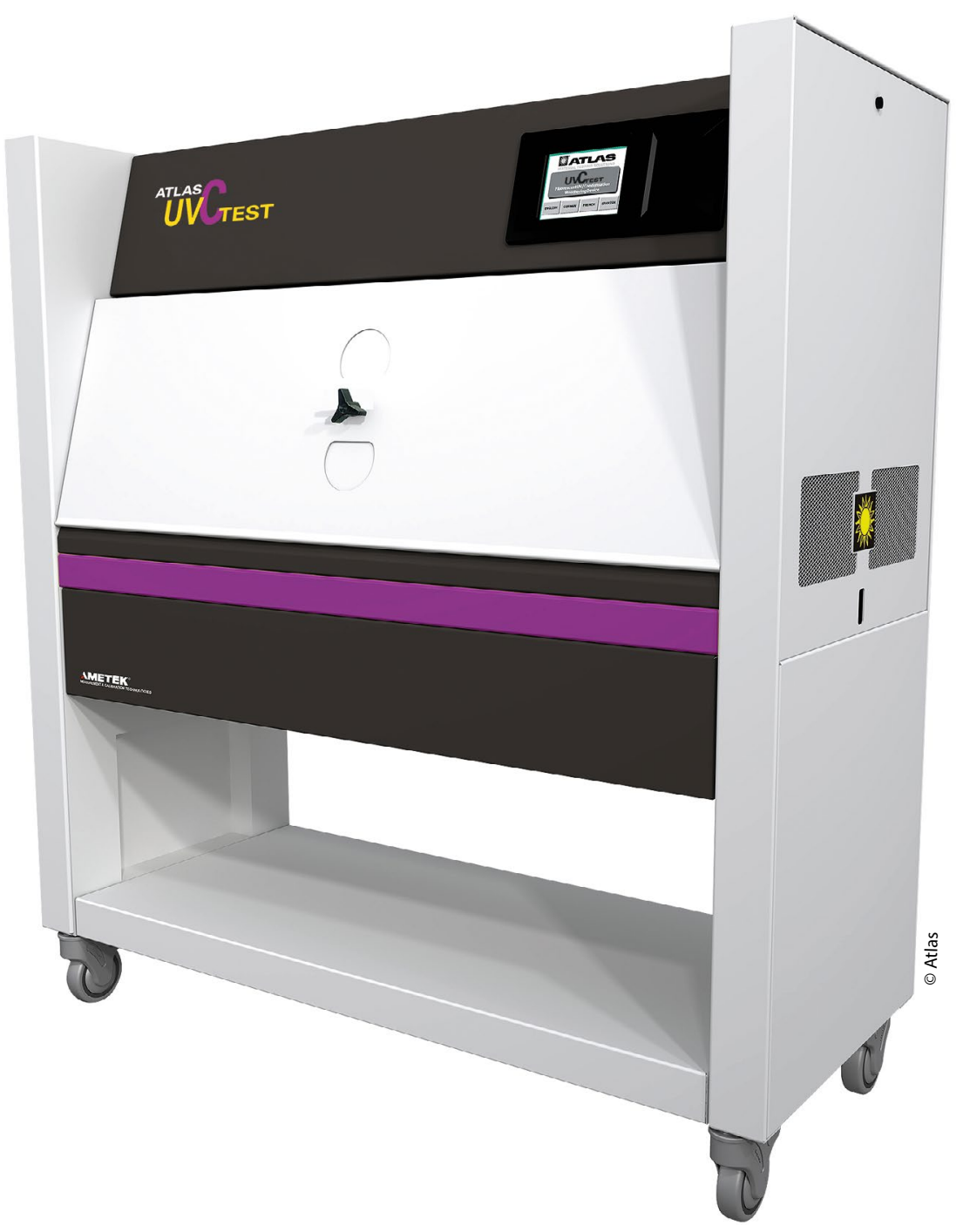

Mit einer auf $254 \mathrm{~nm}$ Wellenlänge zentrierten Bestrahlung lässt sich der Einfluss von Desinfektionsmaßnahmen per UV-C-Strahlung simulieren.
Auf die Desinfektion von Oberflächen, mit denen wir in Berührung kommen, wird aufgrund der aktuellen Corona-Pandemie mehr Sorgfalt verwandt. Neben der Reinigung mit flüssigen Desinfektionsmitteln kommen seit Monaten vermehrt Verfahren zum Einsatz, die Oberflächen in regelmäßigen Abständen energiereicher UV-C-Strahlung aussetzen. Die Desinfektionswirkung von UV-C liegt in der Strahlungsenergie, die so hoch ist, dass sie chemische Bindungen spalten kann und so Erbgut-Moleküle von Viren und Bakterien zerstört.

Die Materialien, aus denen unsere heutigen Produkte und Oberflächen gefertigt sind, besitzen zwar in der Regel UV- und Lichtschutzmittel, diese sind jedoch auf energieärmere Strahlung von natürlichem oder künstlichem Sonnenlicht ausgelegt. Es besteht also die Sorge, dass Produkte, die häufig mit UV-C desinfiziert werden, schneller altern - respektive verblassen, verfärben oder verspröden - und in der Folge ihre erwartete Lebensdauer nicht mehr erreichen.

\section{UV-Testung}

Seit mehr als 100 Jahren werden Tests zur schädlichen UV-Strahlung aus terrestrischem Sonnenlicht durchgeführt (Wellenlängen $\geq 295 \mathrm{~nm}$ ). Da die natürliche Bewitterung in Echtzeit stattfindet, ist das Bedürfnis eines beschleunigten Verfahrens praktisch und kommerziell berechtigt. So werden für die Beschleunigung der Tests künstliche Lichtquellen verwendet. Mit der 


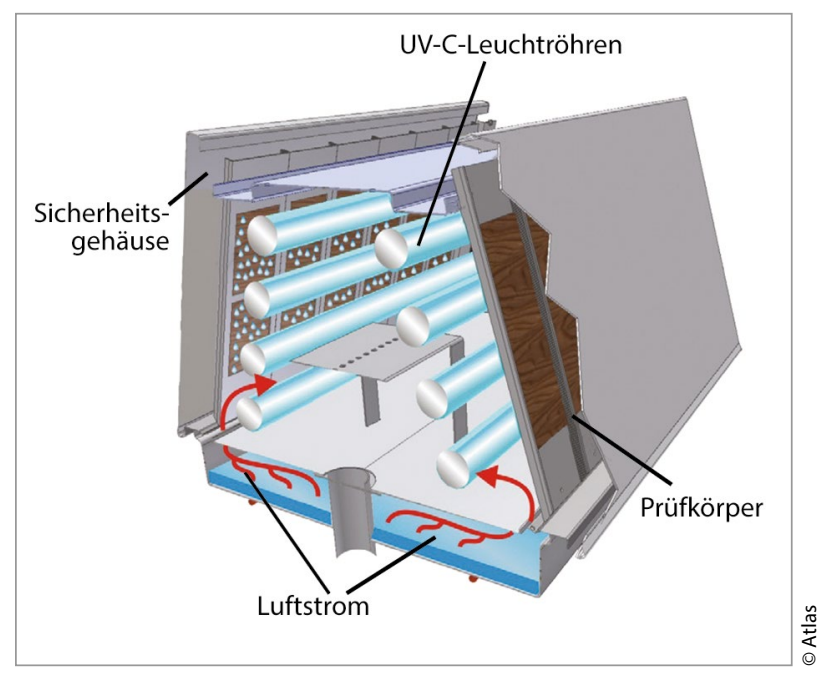

Zeit haben Verbesserungen in Form von Typ der verwendeten Lichtquelle und das Filtern des UV-Bereichs eine immer bessere Simulation des UV-A/UV-B-Sonnenlichts ermöglicht.

Von Forschern wurden die Werkstoffe und jene Additive charakterisiert, die sich auf Wellenlängen $\geq 295 \mathrm{~nm}$ konzentrieren. Die in den Werkstoffen verwendeten Additive wurden also speziell für die in natürlichem Sonnenlicht vorkommenden kritischen Wellenlängenbereiche (295-400 nm) entwickelt, wobei deren Wirkung hinsichtlich UV-C-Strahlung jedoch weitestgehend unbekannt ist. So sind Studien zur Wirkung von UV-C-Exposition auf Werkstoffe - wenngleich sehr aufschlussreich - bis dato nur in begrenztem Umfang verfügbar [1] [2] [3].

\section{UV-C-Exposition nimmt explosionsartig zu}

Seit mehr als 40 Jahren wird UV-C-Strahlung (insbesondere im Zusammenhang mit Lichtquellen mit einer bei $254 \mathrm{~nm}$ zentrierten Spektralleistung) für die Wasser-
Schematischer Aufbau des Testgerätes für eine Alterungsprüfung durch UV-C-Strahlung. aufbereitung aber auch zur Desinfektion - insbesondere in der Pharmazie und Medizin - verwendet [4]. Alle Bakterien und Viren (einschließlich Coronaviren) sind für die UV-C-Exposition empfänglich. Dabei variieren die erforderlichen Dosierungen, um verschiedene Organismen vollständig unschädlich zu machen.

Die Verwendung von UV-C-Quellen zur Desinfektion war ursprünglich auf Operationssäle in Krankenhäusern und in der Arzneimittelforschung beschränkt. Also Orte, an denen Keimfreiheit einen besonders hohen Stellenwert hat. Anfang 2020 nahmen die Forschungen zur UV-C-Exposition zur Verhinderung einer Verbreitung der COVID-19-Infektion explosionsartig zu. Im Fokus stehen unter anderem Transportfahrzeuge und öffentliche Bereiche.

\section{Unkontrollierte Dosierung}

Darüber hinaus sind UV-C-Sterilisatoren nun auch für den privaten Gebrauch online und in ausgewählten Einzelhandelsgeschäften erhältlich. Diese kleineren Geräte sind häufig für die Desinfektion von

\section{Grundlagen der ultravioletten Strahlung}

Der ultraviolette (UV) Teil des elektromagnetischen Spektrums umfasst Wellenlängen von 400 bis $100 \mathrm{~nm}$ und unterteilt sich in drei Bereiche:

- UV-A (315-400 nm) - fast $95 \%$ der Strahlung, die auf die Erdoberfläche trifft. Viele reine Polymere absorbieren diese Wellenlängen und es kommt zu photochemischen Alterungsprozessen

- UV-B (280-315 nm) - der größte Anteil hiervon wird von der Atmosphäre gefiltert, hat jedoch eine höhere Energie, die ebenfalls Polymere schädigen kann

- UV-C (100-280 nm) - gelangt nicht auf natürlichem Weg auf die Erdoberfläche, wird seit Jahrzehnten insbesondere bei der Wasseraufbereitung verwendet

Küchenarbeitsplatten und Elektrogeräten sowie von elektronischen Geräten wie Mobiltelefone, Tablets und Computerperipheriegeräte ausgelegt. Auch von Reinigungsfirmen für Haushalte und Betriebe wird inzwischen UV-C-Desinfektion angeboten. Während also die UV-C-Desinfektion bisher unter relativ kontrollierten Umständen mithilfe von eigens dafür entwickelten Geräten von geschulten Nutzern und in begrenzten (jedoch wirkungsvollen) Dosierungen angewendet wurde, kann im Grunde mittlerweile jeder - auch mit geringen Kenntnissen über die potentiellen Gefahren - Werksstoffe UV-C aussetzen. Aufgrund der Vielzahl der auf dem Markt erhältlichen UV-C-Desinfektionsgeräte ist es zudem schwierig, Details zur tatsächlichen UV-C-Dosierung für jede Anwendungsmöglichkeit in Erfahrung zu bringen. Doch auch bei klaren Anweisungen zu Dauer und Intervallen, die für eine wirkungsvolle Desinfektion erforderlich sind, handeln Nutzer von Privatgeräten teils nach dem Motto „Viel hilft viel“. Dies würde die UV-C-Belastung der bestrahlten Produkte deutlich erhöhen.

\section{Standardisierung der UV-C-Werkstoffprüfmethoden}

Bis dato liegen nur zwei veröffentlichte Test-Standards vor, die sich speziell auf die UV-C-Prüfung von Werkstoffen beziehen. Eine davon ist IEC60335-1 (2020), Anhang T mit dem Titel „UV-C Strahlenwirkung auf nichtmetallische Werkstoffe” und verweist auf den Standard ISO 4892-1 und -2 , der die allgemeinen und Xenonlichtbogen-spezifischen Anforderungen für künstliche Bewitterungstests darstellt [6]. Im Anhang wird auf die Veränderung von 4892-2 zur Verwendung „...einer Quecksilberniederdrucklampe..." mit einer Beleuchtungsstärke von 10W/m² @ 254nm hingewiesen. Weitere Testanforderungen sind eine Schwarzstandard-Temperatur von $63^{\circ} \mathrm{C}$ und einer Expositionszeit von 1000 Stunden.

Hinsichtlich der Durchführbarkeit dieser Methode bestehen einige Bedenken: Erstens ist es unpraktisch (wenn nicht gar technisch unmöglich), kommerziell erhältliche und für die ISO 4892-2 ausgelegte Xenonlichtbogen-Geräte mit UV-C-Lampen $\mathrm{zu}$ modifizieren. Zweitens erscheint die Schwarzstandard-Temperatur für die Simulation der Oberflächentemperatur zu hoch, da typische UV-C-Desinfektionsanwendungen in der Regel in Innräumen bei we- 
sentlich niedrigeren Umgebungs-Temperaturen stattfinden. Letztlich übersteigt auch die Expositionsdauer von 1000 Stunden die typischen Anwendungen bei weitem, selbst wenn UV-C-Geräte über den notwendigen Gebrauch hinaus eingesetzt würden. Um realitätsnahe UV-C-Expositionsdauern $\mathrm{zu}$ erreichen, würden vermutlich weniger als 100 Stunden ausreichen.

Die zweite Methode stammt von der Business and Institutional Furniture Manufacturers Association, dem Verband der Geschäfts- und Institutionsmöbelhersteller (BIFMA) [7]. Neben vielen anderen Tests wird in diesem Dokument die Anforderung erwähnt, eine UV-C-Lichtquelle (mit einer bei $254 \mathrm{~nm}$ zentrierten Strahlung) innerhalb von $291 \mathrm{~kJ} / \mathrm{m}^{2}$ zwischen 12 und 24 Stunden zu verwenden. Diese Testdauer basiert auf einer erwarteten Lebensdauer von sieben Jahren für den exponierten Werkstoff bei einer UV-C-Anwendung pro Woche und einer Dosierung von $0,8 \mathrm{~kJ} / \mathrm{m}^{2}$ UV-C pro Anwendung. Dies entspricht der Anforderung, einen spezifischen Erreger unwirksam zu machen

Die Autoren des BIFMA-Standards haben 2014 logische Annahmen zu UV-C-Anwendungen aufgestellt: Die erforderliche UV-C-Dosierung muss im Hinblick auf die Zunahme der Anwendungsumgebungen für die UV-C-Desinfektion - Dosierung pro Anwendung und die potentielle Anwendungsanzahl pro Woche - weiteren Überprüfungen unterzogen werden.

Entwicklungen von Testmethoden bei globalen Normungsorganisationen, wie ASTM und ISO, stecken zum Zeitpunkt der Verfassung dieses Berichts noch in den Kinderschuhen. Innerhalb mehrerer Organisationen wurden mittlerweile Arbeitsgruppen gegründet, um die Reaktion verschiedener Polymere auf UV-C zu untersuchen und so alle Informationen bereitzustellen, die benötigt werden, die Entwicklung geeigneter UV-C-Prüfmethoden und neuer Standards zu realisieren.

\section{Kommerzielles Testgerät}

Um die wachsenden Marktanforderungen zur UV-C-Testung zu erfüllen, hat Atlas vor Kurzem ein kommerzielles Gerät eingeführt. Das Testgerät ist darauf ausgelegt, die Haltbarkeit von Werkstoffen zu testen, die UV-C-Strahlen (auf $254 \mathrm{~nm}$ zentriert) ausgesetzt werden. Es basiert auf der UVPrüfung mit Leuchtstoffröhren/UV-Plattform, verfügt jedoch im Hinblick auf die Sicherheit und Zuverlässigkeit über besondere Modifikationen. So simuliert die Intensität der UV-C-254-Lampen die ultraviolette keimtötende Bestrahlung (UVGI), wie sie von vielen UV-C-Desinfektionsgeräten verwendet wird. Das Gerät kontrolliert dabei sowohl die Bestrahlungsstärke als auch die Schwarzstandardtemperatur. Hell- und Dunkelphasen lassen sich ebenfalls programmieren.

Um ein besseres Verständnis für die für die verschiedenen Anwendungen erforderliche UV-C-Dosierung zu erlangen, hat Atlas auch einen einfach zu bedienenden UV-C-Dosierungsrechner entwickelt. Hierfür sind folgende Eingaben erforderlich:

- UV-C-Dosierung pro Anwendung (die je nach verwendetem UV-C-Desinfektionsgerät variieren können)

- Anzahl der erwarteten Anwendungen pro Woche

- Erwartete Lebensdauer des geprüften Werkstoffs

- Strahlungsintensität während des Tests

- Dauer von hellen und dunklen Phasen (optional)

Auf dieser Basis bestimmt der Rechner die Anzahl der Expositionsstunden, die erforderlich wären, um die gleiche Menge an UV-C-Dosierung zu erreichen, die der Werkstoff in der Serviceumgebung der Endnutzung erhalten würde.

\section{Fazit}

Viele Polymere sind zwar mit Additiven zum Schutz vor natürlichem Sonnenlicht gewappnet, dagegen sind Additive für den Schutz gegenüber UV-C-Strahlung noch weitgehend unbekannt. So bereiten die Auswirkungen von UV-C-Strahlung auf die Materialien und Produkte unseres alltäglichen Lebens Sorge hinsichtlich des Aussehens oder des vorzeitigen Versagens. Die gegenwärtigen Prüfmethoden müssen auf die heutigen Anwendungen - insbesondere die Desinfizierung per UV-C-Strahlung - weiter verbessert werden. Kommerzielle UV-CPrüfgeräte unterstützen diese Aufgabe. //

\section{Literaturhinweise}

[1] Lindsley, et al., "Effects of Ultraviolet Germicidal Irradiation (UVG) on N95 Respirator Filtration Performance and Structural Integrity", Journal of Occupational and Environmental Hygiene, 12: 509-517, (2015).

[2] Wintzer, Walther, and Leuthaeusser, "Studies on UV-C Treatment of Polyamide Fibers for Improved Adhesion on tup and TPA," Journal of Chemistry and Chemical Engineering 9, 38-44, (2015).

[3] Irving, et al., "A Comparison Study of the Degradative Effects and Safety Implications of UV-C and $405 \mathrm{~nm}$ Germicidal Light Sources for Endoscope Storage," Polymer Degradation and Stability 133, 249-254, (2016).

[4] IUVA Fact Sheet on UV Disinfection for COVID-19, www.iuva.org

[5] van Doremalen, et al., "Aerosol and Surface Stability of HCoV-19 (SARS-CoV-2) Compared to SARS-CoV-1,"The New England Journal of Medicine, 10.1056/NEJMc2004973, (2020).

[6] IEC 60335-1: 2020, Household and similar electrical appliances - Safety - Part 1: General requirements

[7] BIFMA HCF 8.1-2014, Health Care Furniture Design - Guidelines for Cleanability

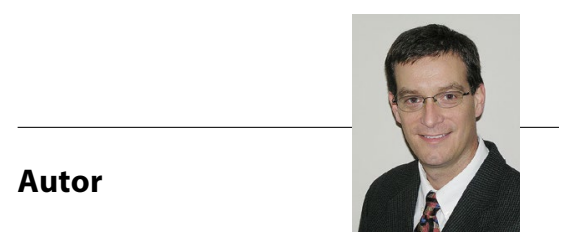

\section{Matthew McGreer}

Senior Produktmanager

Atlas Material Testing Technology LLC

Illinois, USA

matt.mcgreer@ametek.com

www.atlas-mts.de 


\section{Bauteilsauberkeit stabil sichern}

\section{Prozessflüssigkeiten überwachen}

\section{Tensidkonzentration - Badverschmutzung}
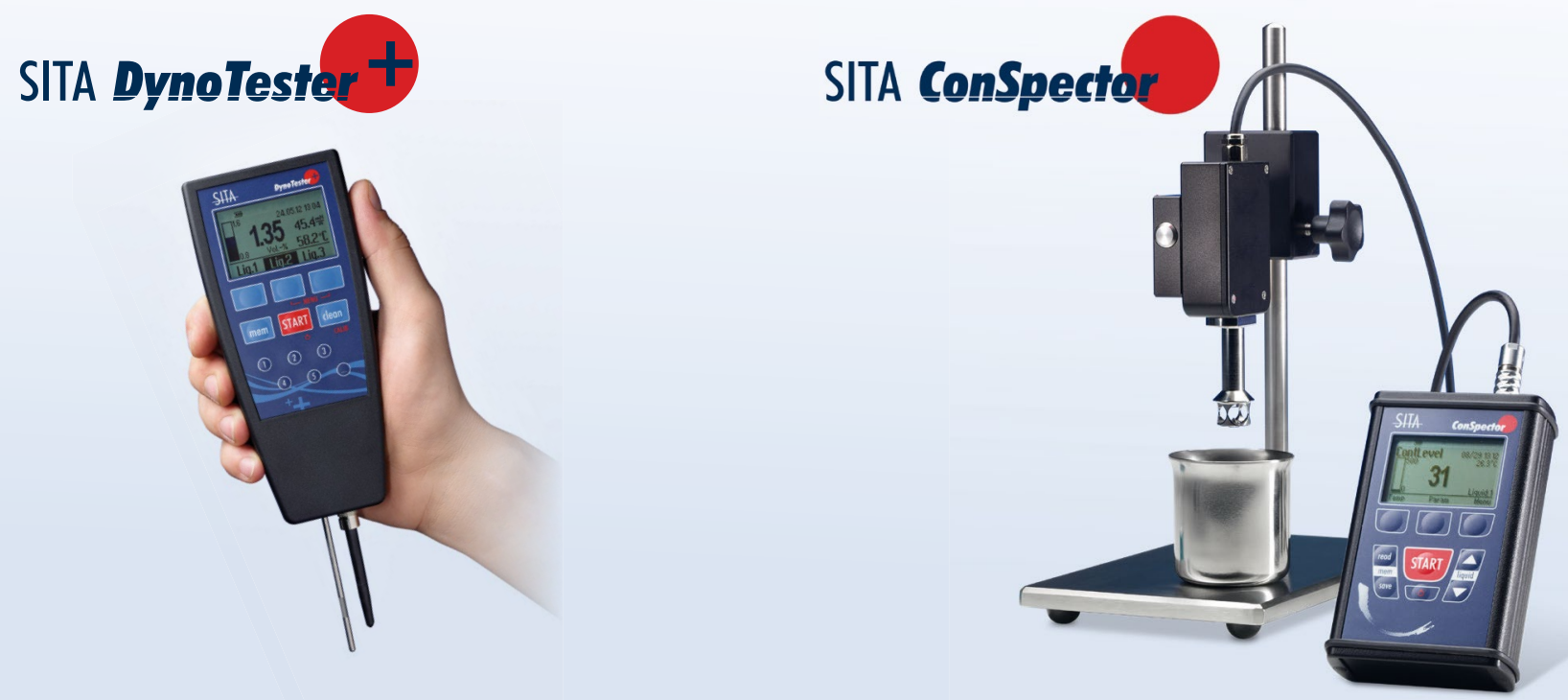

Bauteiloberflächen kontrollieren

Teilesauberkeit - Oberflächenbenetzbarkeit

SITA CleanoSpector

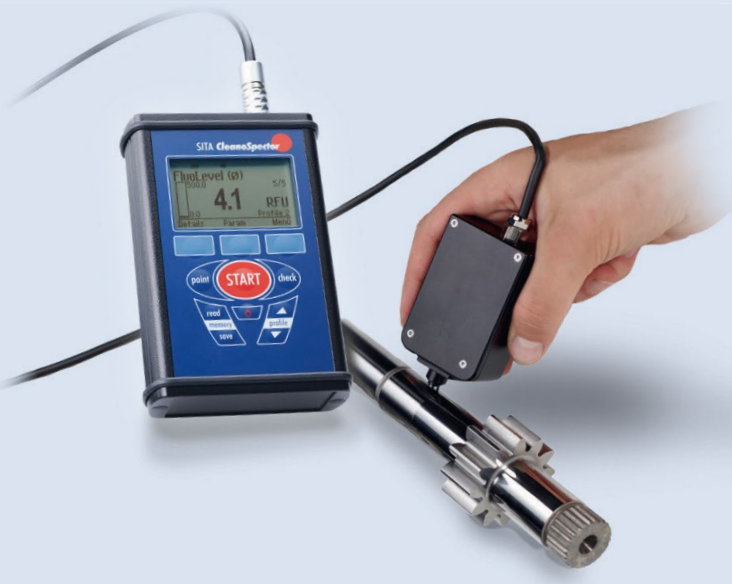

SITA Surfaspector

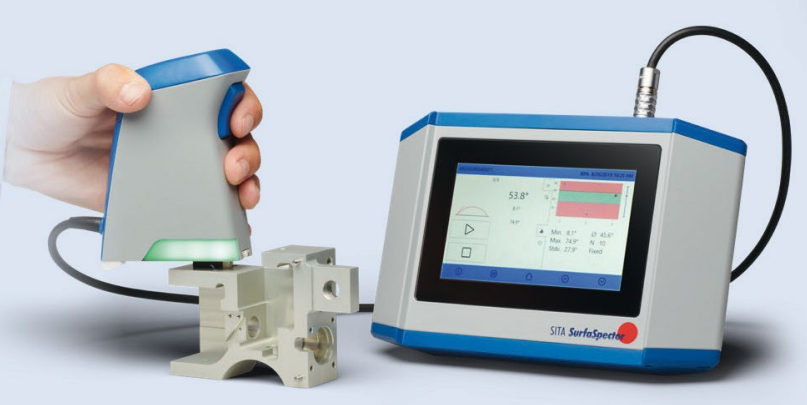

SITA Messtechnik GmbH

Ihr Partner für qualitätssichernde Prozessführung 